\title{
Access to Health Care for Individuals With Developmental Disabilities From Minority Backgrounds
}

\author{
Amanda Reichard, Therese Marie Sacco, and H. Rutherford Turnbull, III
}

\begin{abstract}
In this project we examined access to health care by individuals with developmental disabilities in Kansas from low-income populations and from minority backgrounds. Four criteria for determining access were employed: availability, accessibility, affordability, and appropriateness of care. Factors that pose barriers and that facilitate access are described and recommendations are set out, with particular reference to the 2002 Report of the Surgeon General of the United States, related to health status of people with mental retardation.
\end{abstract}

The literature documents that people with developmental disabilities experience greater difficulty in securing appropriate and affordable health care than do individuals without disabilities (Vittek et al., 1994; Ziring et al., 1988), but the care needs of the former population often include more than basic medical care (Rosenfeld, 1994). The 2002 U.S. Surgeon General's Report on Health Disparities and Mental Retardation concluded, with respect to the population of individuals with developmental disabilities that:

Compared with other populations, individuals of all ages with mental retardation experience poorer health and more difficulty in finding, getting to, and paying for appropriate health care. These challenges are even more daunting for people with mental retardation from minority communities with many cultures and languages and whose culture and language may not be reflected in available health services. As with many other disabling conditions, the multiple disorders associated with mental retardation are found disproportionately among low-income communities that experience social and economic disparities when they seek health care. Developmental disabilities compound these disparities because many health care providers and institutional sources of care avoid patients with this condition. Without direct clinical experience, health care providers may feel incapable of providing adequate care. They may not value people with mental retardation and their potential contributions to their own health and to their communities (U.S. Department of Health, 2002, p. xii).

Our research confirms these conclusions and is organized according to the same criteria we used in reporting about access to dental care for individuals with developmental disabilities in Kansas (Reichard, Turnbull, \& Turnbull, 2001): (a) availability, (b) affordability, (c) accessibility, and (d) appropriateness of care, all reported by the individuals themselves or by their proxies.

With regard to availability, previous research documents the insufficient numbers of primary care physicians (Kindig \& Yan, 1993; Knapp, Paavola, Maine, Sorofman, \& Politzer, 1999; Politzer, Harris, Gaston, \& Mullan, 1991; Rabinowitz, Diamond, Hojat, \& Hazelwood, 1999), especially in rural areas (Connor, Hillson, \& Krawelski, 1995) and communities with a large number of minority citizens (Caudle, 1993; Cornelius, 1993; Friedman, 1994; Giachello, 1998; Kindig \& Yan, 1993). There are insufficient numbers of dentists, especially for children (Brooks \& Dwyer, 1997; Nainar, Edelstein, \& Tinanoff, 1996; Waldman, Perlman, \& Swedloff, 1998) and most especially for those in rural (Waldman, 1995) and innercity (Waldman, 1994) areas.

Physicians themselves report that they are not prepared to treat individuals with developmental disabilities (Darling \& Peter, 1994; Reichard, Turnbull, \& Turnbull, 1999; F. Scott, 1990; F. G. Scott, Lingaraju, Kilgo, Kregel, \& Lazzari, 1993), in part because of insufficient training (Levy \& Hyman, 1993). Dentists also report insufficient training (Academy of Dentistry for Persons With Disabilities, 1996; Burtner \& Dicks, 1994; Fenton, 1993); dentists who did have substantial training experiences with individuals having developmental disabilities indicated a willingness to treat those individuals (Ferguson, Berentsen, \& Richardson, 1991; 
Stiefel, Truelove, Martin, \& Mandel, 1997). Further, they believed that their practice benefited from accepting patients with developmental disabilities (Ferguson et al., 1991).

Family reports indicate that a limited number of physicians and dentists will treat individuals with developmental disabilities (Brooks \& Dwyer, 1997; Nainar et al., 1996; Reichard, Turnbull, \& Turnbull, 1999; Simons, 1987; U.S. Department of Health, 2002), especially those whose only source of payment is Medicaid (Brooks \& Dwyer, 1997; Nainar et al., 1996) or those who are women seeking gynecologic services (Kopac, Fritz, \& Halt, 1998). The causes include low Medicaid reimbursement rates (Burtner \& Dicks, 1994; Damiano, Brown, Johnson, \& Scheetz, 1990; Lang \& Weintraub, 1986; McKnight, Myers, \& Dushku, 1992; Nainar \& Tinanoff, 1997; Venezie \& Vann, 1993); the requirement of prior authorization for treatments (Venezie \& Vann, 1993); challenges in coordinating service, the increased time required to treat without increased reimbursement, and excessive paperwork (Diamond \& Zarafu, 1988; Hughes, Halfon, Brindis, \& Newacheck, 1996; Palfrey, Samuels, Haynie, \& Cammisa, 1994; Reichard \& Turnbull, 2002; Reichard, Turnbull, \& Turnbull, 2001); the "Medicaid stigma" (Davis et al., 1999; Lam, Riedy, \& Milgrom, 1999) as well as patients' history of missing or breaking appointments (Damiano et al., 1990; Lang \& Weintraub, 1986; Venezie \& Vann, 1993) and failing to comply with posttreatment regimens (Academy of Dentistry, 1996). Finally, there are transportation barriers (Rask, Williams, Parker, \& McNagny, 1994; Smith, Kreutzer, Goldman, Casey-Paal, \& Kizer, 1996) to short-term care (Crain, Kercsmar, Weiss, Mitchell, \& Lynn, 1998) and to specialized services, especially when the patients live in rural or remote areas (Harbaugh \& Smith, 1998).

Accessibility is the second barrier (Grabois, Nosek, \& Rossi, 1999), especially for women (Welner, 1998), patients who do not speak English (Perkins, Simon, Cheng, Olson, \& Vera, 1998; Woloshin, Bickell, Schwartz, Gany, \& Welch, 1995), and patients whose culture differs from that of providers (Perkins et al., 1998; Riddick, 1998; Vasquez \& Javier, 1991; Woloshin, et al., 1995).

The third barrier is affordability. High poverty rates among adults and children with mental retardation mean that a large proportion of them rely on publicly financed health care insurance, which does not always serve their needs (U.S. Department of Health, 2002), reimburses providers insufficiently, and is replete with administrative barriers. Moreover, patients lack the ability to pay out-of-pocket, even when some insurance reimbursement is available (Bolden, Henry, \& Allkian, 1993; Kopac et al., 1998); face lifetime spending caps and narrow eligibility standards (Hughes et al., 1996; Palfrey et al., 1994); and become disentitled to Medicaid when they reach the age of majority (Hughes et al., 1996; Palfrey et al., 1994; Umbarger, Turnbull, Morningstar, Reichard, \& Moberley, 2001).

Finally, inappropriate treatment based on disability arises in part from the reluctance or incompetence of providers to consider parents' perspectives (Darling \& Peter, 1994; Simons, 1987; Wilson, 1994) and from the lack of structural and communication accommodations (Barnett \& Ziring, 1988; Ebert \& Heckerling, 1995; McEwen \& Anton-Culver, 1988; Nosek et al., 1995; Ralston, Zazove, \& Gorenflo, 1996; Welner, 1998). Attitudinal barriers also persist (Brooks \& Dwyer, 1997), with screening-out, scheduling limitations, and treatment in segregated settings manifesting bias (Grabois et al., 1999; Reichard et al., 2001). Because some physicians have not been trained in special health concerns of women with physical disabilities, these women have an increased risk for gynecological malignancies (Nosek et al., 1995; Welner, 1998).

Cultural insensitivity remains a considerable obstacle to obtaining health care for many minorities (Brookins, 1993; Chestnut, 1994; Cornelius, 1993; Fishman, Bobo, Kosub, \& Womeodu, 1993; Giachello, 1998; Nelkin, 1996), despite the importance of considering culture in the diagnosis and management of disease (Brookins, 1993; McCubbin, Thompson, Thompson, McCubbin, \& Kaston, 1993). Moreover, racism affects treatment access (Cornelius, 1993; Friedman, 1994; Giachello, 1998; Giambruno, Cowell, Barber-Madden, \& Mauro-Bracken, 1997; Horner, Oddone, \& Matchar, 1995; Kiefe \& Hyman, 1996; King \& Brunetta, 1999; Kogan, Kotelchuck, Alexander, \& Johnson, 1994; Mort, Weissman, \& Epstein, 1994; Nelkin, 1996; Newacheck, Hughes, \& Stoddard, 1996; Perkins, 1999; Williams, 1999).

\section{Method}

Participants

All 23 of our research-respondent families had a child with a developmental disability and an in- 
come below 200\% of the federal poverty level for that family or individual. Disabilities included mental retardation (57\%); seizures (35\%); cerebral palsy $(30 \%)$; other health conditions, such as heart or kidney problems (26\%); chromosomal syndromes, such as Down and Williams syndromes (22\%); and degenerative diseases, such as cystic fibrosis and muscular dystrophy (9\%). Among these families, most (39\%) were Caucasian; 26\% each, African American and Hispanic; and 9\%, American Indian. The majority $(52 \%)$ were between 6 and 18 years old, $35 \%$ between 19 and 49 , and $13 \%$ between 0 and 5 . The slight majority (56\%) of the individuals with developmental disabilities were male, and all respondents were distributed nearly equally across the state of Kansas.

We recruited these 23 families by contacting and asking representatives of eight agencies across the state to identify families who met our criteria. We acknowledge that there may be bias in the sample, because the only families willing to talk with us may be those who are relatively satisfied with their health care.

\section{Data Collection}

We used qualitative interviewing techniques to collect data (Berg, 1998; Marshall \& Rossman, 1995), following the protocols that apply especially to families from diverse backgrounds (Jarrett, 1993; O'Brien, 1993). We interviewed each family twice for 1 to 1.5 hours each time. In the first interview, we sought information about as many health care topics as possible, by asking broad, open-ended questions and encouraging respondents to talk about their experiences. We guided conversation with prompts, asking new questions during lulls, and redirecting the respondent when the discussion veered from topics not related to health care. In the second interview, we asked families to clarify and expand their first interview responses where necessary, and we encouraged discussion of topics omitted from the first interview.

\section{Data Analysis}

Our analysis was transcript-based; two researchers were required to jointly review field notes and summarize debriefing sessions. The researchers separately analyzed interview transcripts, not just once but twice. In all data analysis we followed the established protocols for qualitative research, including generating categories and codes, establishing trustworthiness, assuring triangulation, using peer debriefing and negative case analysis, conducting member checking, and controlling for dependability and confirmability (Krueger, 1994; Stewart \& Shamdasani, 1990; Taylor \& Bogdan, 1998). We used The Ethnograph, a computer software program, to help organize and retrieve interview data (Seidel, Friese, \& Leonard, 1995).

\section{Findings}

The conceptual framework for this research consisted of the "Four A" inquiry that we used in our report on dental care in Kansas for individuals with developmental disabilities (Reichard, Turnbull, \& Turnbull, 2001): Is health care-medical, OB/GYN, and dental-(a) available, (b) accessible, (c) affordable, and (d) appropriate? This framework allows us to discuss findings related to OB-GYN care, auxiliary services, and the health care system in general.

\section{Availability}

Under the category availability, we inquired whether health care is available (i.e., does it exist?). Subsumed within this category are questions related to (a) sufficient providers both who can and will give appropriate care, including availability by phone and for appointments when necessary; (b) what coordination assistance is available; and (c) transportation and distance to appointments.

Provider availability. Every family except one had easy access to a primary care provider for their child. Several said that they were fortunate to have a physician who accepted Medicaid; some had encountered primary care physicians who did not accept Medicaid patients. Many families in a rural farming and meat-processing community believed that there were no doctors in the area who were knowledgeable about treating individuals with disabilities. A few parents reported difficulty finding trustworthy practitioners to serve their child with disabilities. Most families had been able to locate all of the health care professionals they needed, including physicians, dentists (for children only), specialists, and auxiliary health professionals, although some had to continue seeking out new providers until they found competent professionals who were willing to treat their children.

Many families, especially those in rural areas, noted that they must travel long distances to access specialist care. For those not living in rural areas, however, access to specialists posed no problems. 
Several families noted that their physicians (general practitioners and specialists alike) were readily available by phone, and most families could make appointments for their children when they needed them. Although nearly all families said that they had to wait long periods to see their child's physician once they arrived at the providers' offices, the waits were not due to their child's disabilities; they did not wait any longer than did patients without disabilities.

Availability of dental care differed by age. For younger individuals with disabilities, the ease of finding a dentist varied. Some had no problems whereas others (a) had to travel, (b) had to wait a long time, (c) could not afford it, or (d) could not find a dentist who would accept Medicaid. For many adults, availability of dental care "takes a back seat to" affordability because Medicaid does not cover their treatment.

Coordination of health care. Because none of the families received formal assistance in coordinating their child's health care, many had to spend much time coordinating care. One family had no formal case manager but, instead, received support from a network of friends, including those who, because of their professional status, know how to secure appropriate care from qualified providers.

Information and referral systems. Most families located their health care professionals through the recommendations of other families who had a child with disabilities or from other health care providers. Some families located providers through the phonebook, suggestions from their child's teachers, or through a family-support worker. In addition, community-based developmental disability organizations, social workers, teachers, and others provided information on where to find the services families needed.

Distance and transportation. Most families lived close to primary care providers. For specialist care, however, many of those outside of the Kansas City or Wichita metropolitan areas-namely, families living in rural areas-had to travel long distances to receive specialist care. Most supplied their own transportation or found other means (i.e., transportation service, taxi, family member, friend). Families in rural areas, however, spoke of the difficulties in traveling to distant urban areas for specialist care. For example, one father reported that he got no sleep for 2 days when he took his daughter to the city because he could not afford to miss more than one day of work.
Continuity. Some families switched physicians because they were unhappy with some of the doctor's practices. Others switched when they did not choose to do so, sometimes because the physician or dentist retired, quit the practice, or refused to accept Medicaid. Families remained with their primary care physician for many reasons, including (a) trust, (b) good bedside manner, (c) affordability or payment plan, and (d) willingness to refer. Many stayed for the sake of continuity, which allowed them to (a) avoid explaining the child's history repeatedly, (b) avoid moving records, and (c) improve the child's health. A few families had been with providers for long periods, even generations. Some families remained with their current dentist because they were pleased (e.g., good treatment, trust, good with individuals who had disabilities) or because they believed they had few choices.

\section{Accessibility}

Accessibility relates to physical and linguistic issues. Most families did not confront accessibility challenges. Overall, providers' offices were accessible; however, 2 families mentioned difficulties with the parking lots and 3 families said that examination rooms did not accommodate wheelchairs. Moreover, several families indicated they had been unable to make necessary health care accommodations in their homes (e.g., adding ramps, enlarging door frames, and installing bathroom equipment) because they could not afford the out-of-pocket expense or were unable to obtain alternative funding.

\section{Affordability}

Affordability relates to whether families are able to purchase health care. We asked whether the families had some form of health insurance and, if so, whether the insurance provided sufficient coverage of necessary services. We also asked them to list their out-of-pocket expenses.

Most families received Medicaid as their primary health insurance. Others had Medicaid and private health insurance that picked up coverage where Medicaid left off. (In Kansas, a family who meets all eligibility requirements for Medicaid coverage can also maintain private health insurance coverage). Only a few had neither of these or alternated between having and not having coverage because their incomes changed frequently and sometimes exceeded the eligibility guidelines. A few families obtained funds from alternative sources, such as Indian Health Services, Kansas Crippled 
Children's Prescription Health Services, and statesponsored supported living grants. Those families without insurance paid for services out-of-pocket or simply did not receive the needed service.

Some families described satisfaction with Medicaid's extra benefits and flexibility. Others discussed their problems, especially SSI and Medicaid. Because of income limitations and their own income variability, families sometimes needed SSI-Medicaid coverage and sometimes did not. Not knowing from month to month whether they had coverage was stressful emotionally and burdensome financially because, without coverage, families had to pay out-of-pocket in those months when they made even a small amount over the income limit. (That consequence obtained at the time of the research. Since then, Kansas Medicaid has changed its format to ensure continuous eligibility for one year.) In addition, the Kansas Medicaid plan limited reimbursement for dental care, which stopped at age 21, causing families of adults to pay out-of-pocket for dental care that was not otherwise reimbursable by an insurance carrier (Reichard, Turnbull, \& Turnbull, 2001).

The utility of private insurance varied for those who had it. Those who could afford private insurance paid high rates for coverage. For 3 families, private insurance served them well. Even with high rates, however, coverage from such insurance was sometimes insufficient for meeting the needs of the person with a disability. For some families, coverage was inadequate to meet their child's needs, particularly dental care. For still other families, private insurance was completely unaffordable. Others found ways to afford it, despite high rates, co-payments, and deductibles.

Several families discussed bureaucratic inconveniences of funding in general as well as Medicaid and private insurance. They reported that the first insurance company response was to "deny coverage." One respondent said that she had to be aggressive when seeking coverage: "If you don't push, you have to pay out-of-pocket." Referring to Medicaid dental coverage, another respondent said, "Sometimes it's easier to just borrow the money or beg, borrow, or steal it to pay for it than it is to go through the red tape."

\section{Appropriateness}

Appropriateness relates to whether the health care meets the individual's needs. Criteria for appropriateness include (a) whether health care needs are met, (b) how families define quality health care, and (c) appropriate and inappropriate characteristics of providers. (It is important to note, however, that families and medical professionals may have differed in their definitions of appropriateness.)

Health care needs met. Most families reported that health care needs of their child with developmental disabilities were met, especially for medical care. However, when asked what needs other than medical care were not met, several families cited dental care, orthodontic care, accessible equipment, counseling, and structural accommodations in the home.

Definition of quality. Above all else, most families defined quality health care as having practitioners who were warm, caring, patient, and respectful of individuals with developmental disabilities. In addition, many families reported that quality health care included practitioners who provided effective treatment for individuals with developmental disabilities; knew how to "work with" parents, especially in giving referrals when necessary; were straightforward and honest; and were diligent in attempting to solve health-related problems. Some families also said that quality included available physicians, choice of providers, continuity of care, and ease in obtaining appointments.

Several parents reported accommodations that, in their judgment, increased the appropriateness of health care services. For example, some families indicated that several physicians or their staff members accommodated needs above and beyond what they considered good quality treatment. Two physicians were even known to "go to bat" for children by meeting with or writing letters to teachers or administrators about diagnoses and the need for school staff to follow specific routines to ensure children's well-being. Similarly, one family described a hygienist who worked hard to keep their child calm while he was at the dentist's office. In addition, some families stated that they have received financial adjustments from physicians or dentists, either through payment plans or reduced fees.

Characteristics and practices that families regarded as inappropriate included (a) poor quality or incorrect medical treatment, (b) not taking time with the child, (c) not being respectful of parent and child, (d) poor communication practices, and (e) having too little information about their child's disability.

Several families discussed their experiences 
with physicians who disclosed relevant health information about their child in a very untimely manner. In several instances, the delay in information resulted from doctors not knowing the cause of the problem; in other cases, doctors did not fully share all information they had. For a few families, a delay in diagnosis was detrimental to their child's health. For example, one boy's delay in accurate diagnosis prevented him from receiving the early heart treatment he needed.

\section{Obstetric and Gynecologic Care}

A wide range of experiences prevented access to OB/GYN care for the 5 women in this study who indicated a need for this specialty or related care. These individuals lived in all areas of the state, and their disabilities ranged from developmental disabilities to cerebral palsy to mental retardation and seizures. Three were in their 20s and 2 were teenagers.

Frequency of OB-GYN care. Two women (1 with learning disabilities and developmental disabilities, 1 with cerebral palsy and hydrocephalus) regularly received this care. Two others received one-time involuntary pelvic exams while under anesthesia for other treatments; they did not, however, receive exams regularly, either because the examination itself was too invasive, given the requirement of general anesthesia for the patient, or because, according to the family member (always a mother) or the patient's physician, it was not necessary because the women were not sexually active.

Yet another woman (the 5th respondent) needed gynecologic care (according to her mother) but had never received it, apparently because her mother assumed the physician provided it routinely or because she had never explicitly made her wishes known to the doctor. Indeed, this woman's sister and mother did not want to have to ask for a PAP smear and breast examination. The mother and sister believed, however, that the patient needed these examinations but did not think that there was anyone in their home town who would do it and that they would have to take the patient to the closest city and deal with a new physician.

Particular procedures. One of the 5 women had had an involuntary tubal ligation. For this procedure, she was under anesthesia; during the procedure, the gynecologist conducted a pelvic examination, too. He believed it would be too traumatic for the patient to have another pelvic examination without anesthesia. Except for the one pelvic ex- amination, then, the patient had no regular pelvic exams. Moreover, her mother, after consulting with the physician's assistant, concluded that she would not treat her daughter for breast cancer if such a treatment became necessary; therefore, she did not want her daughter to experience a breast or a pelvic examination. The mother also did not plan to continue regular pelvic or breast examinations. Despite her own determination to not have her daughter examined or treated, this mother said she was satisfied with the care her daughter had received from the OB/GYN.

Sexual inactivity as a determinant. Another mother said her daughter did not need the care of an $\mathrm{OB} / \mathrm{GYN}$ practitioner because her daughter was not sexually active. She based this on information given to her by her own gynecologist, who told her that because the daughter was not sexually active, "there isn't really any strong need for a PAP test." According to this mother, however, if she requested a PAP test, the physician would perform the procedure, but her daughter would require general anesthesia. The mother stated her perspective as follows:

I don't really, someday it's probably maybe going to get to be like, as she gets older, maybe if she started having trouble with her periods or something, you know, then she would need it. But, right now, gynecological things are something that we really haven't gotten too much into.

The daughter, however, had had a pelvic and PAP smear procedure while under general anesthetic for another procedure; these OB-GYN examinations were conducted for the mother's own "peace of mind." This mother said she would trust her own gynecologist to "make it the least traumatic for her (daughter)" and "get the job done" should the daughter need that kind of care.

Source of care. One of the 5 women began puberty at age 5 . She started her menstrual cycles at age 9 and began seeing an $\mathrm{OB} / \mathrm{GYN}$ at that point. At the time of our interview, she was seeing her "regular doctor" who "takes care of all that" because her mother wants "one person to take care of the whole thing."

Another 1 of the 5 women went to the publichealth department to receive gynecological care. She liked to go there because the doctor treated her like everyone else, was available to answer questions between visits, and because the health department accepted Medicaid reimbursement. The mother said 
her daughter was pleased with the care there and seemed to understand its importance.

\section{Auxiliary Services}

The auxiliary services included mental health services, having prescriptions filled, case management, nursing, various therapies, psychological and counseling, community-based habilitation services, vocational rehabilitation, and wraparound services. It appeared that little middle ground exists for families regarding auxiliary services. Either they received full, very satisfactory support with which they were pleased, or the services they received lacked quality and caused them to be dissatisfied. Moreover, some families noted having unmet needs in this area, specifically with counseling (3 families), but also with case management, finding accessible equipment, hospital advocate, home care, and respite care. The reasons given for the inability to access services included problems with funding, lack of information regarding how to access the services, and a complete lack of the desired resource.

\section{Health Care System Overall}

Many families talked about how the system has changed over the years. Some noted negative changes. For example, one family described how one special needs group (which the family did not identify) "seems like it disappeared," indicating that the family can no longer access the service. Another family discussed how the Home and CommunityBased Services (HCBS) Waiver once covered family members to care for their child with developmental disabilities, but that it no longer did.

Others noted positive changes. For example, one family said that access to health care had improved over the years because doctors had become more accessible and wait times in offices have become shorter. Another family mentioned that Medicaid has changed to cover more medicines. Some families described how the system accommodated them. For example, one hospital supplied medication for the child at a cost to the hospital, not the family, because Medicaid does not reimburse for that particular medicine. In fact, the hospital mailed the medication to the mother twice a year. In addition, a local health department did not refuse treatment when the family was unable to pay and accepted Medicaid when the family qualified for that benefit.

\section{Discussion and Recommendations}

In light of the previously published literature and the Report of the Surgeon General (2002) related to families from low-income and diverse backgrounds, one may have expected that the Kansas families we interviewed would have faced formidable obstacles to the availability, accessibility, affordability, and appropriateness of health care for their child. Our data reveal, however, that overall, the families have had little difficulty with respect to these four criteria of health care access. Indeed, most have experienced few problems locating the providers they need. In addition, once they find suitable providers, families typically continue under their care as long as possible. Although families often spend much time coordinating their children's care, no one mentioned difficulties with care coordination. Also, for most families, transportation posed no barriers to their children in receiving health care. Nevertheless, families reported challenges that warrant change and improvement in the four criteria areas and in other areas as well: (a) availability, (b) accessibility, (c) affordability, (d) appropriateness, (e) OB/GYN care, and (f) the health care system overall.

\section{Availability}

Some physicians and dentists refuse to accept patients whose only source of payment is their Medicaid benefit. What changes seem warranted? As we indicated in our earlier research about access to dental care (Reichard, Turnbull, \& Turnbull, 2001), changes in regulations and provider reimbursements under Medicaid are necessary so that physicians and dentists will not risk financial loss by treating Medicaid recipients. Second, innovative updates in preand inservice curricula may blunt provider bias against Medicaid patients. Medical and dental schools (preservice) and societies (inservice) could (a) lead by example, encouraging faculty and staff to demonstrate acceptance of Medicaid recipients; (b) require courses that encourage tolerance of and respect for Medicaid recipients (and other differences such as disabilities); and (c) provide increased exposure to a large diversity of populations, including Medicaid recipients. Finally, the state medical and dental societies could strongly encourage their respective members to treat a minimum percentage of Medicaid or other indigent patients pro bono, a model that exists within bar associations. Whether the state as a licensing authority could require prac- 
titioners to treat a mandatory minimum of Medicaid patients or do other pro-bono work is a debatable and rather radical prospect, but it should not to be ruled out. Our recommendations, which are consistent with those of the Report of the Surgeon General (2002), are goals related to increasing providers' knowledge about mental retardation and ensuring that knowledge is disseminated; improving the quality of health care by, among other things, identifying priority areas for quality improvement; developing standards of care; and training health care providers in pre- and inservice forums.

Not surprisingly (given the natural concentration of specialists near tertiary care facilities), families reported difficulty in accessing specialists in rural areas. Fortunately, several potential partial solutions are available: telemedicine, regular rotation of specialists into rural areas and their admission to community hospitals, and in-service training of established general-practice physicians (with an emphasis on the particular issues related to treating patients with developmental disabilities). These potential partial solutions relate to two matters: proper education and provider distribution. Medical and dental schools should continue increasing and improving what they teach and how they expose students to issues of individuals with disabilities. Beyond this, schools and others should encourage some medical and dental students to specialize in the care of individuals with disabilities. In addition, advocates, schools, and community builders must continue encouraging and enticing practitioners to locate in rural areas.

\section{Accessibility}

Our data suggest that most issues of accessibility have been removed. The two largest accessibility barriers were parking lots and home accommodations. State and local medical and dental societies can alert their members to the Americans With Disability (ADA) requirements; local centers for independent living and other disability-advocacy organizations can offer technical assistance related to access; and the State Department of Social and Rehabilitation Services can advocate for a larger budget for home modifications and, through community developmental disabilities organizations, alert families that home-modification funds are available on a one-time basis (with no obligation for repayment).

\section{Affordability}

Generally, families who have Medicaid, whether solely or in combination with private insurance, have their funding needs met; as we have reported, however, there are some exceptions. This finding seems somewhat contrary to the wide-sweeping assertion in the Report of the Surgeon General (2002) that health care financing - in both the public and the private sectors-is deficient. On closer examination, however, our data concur with those of the report. For Kansas families, the problems lie in (a) qualifying for Medicaid, (b) lack of dental care funding for those over 21, (c) gaining information for obtaining funding for additional needs, and (d) processing claims. Similarly, in the report the surgeon general cited publicly financed health care insurance as "not always well-adapted to serving [the] needs" of the "large portion" of those with mental retardation who rely on the public-reimbursement system, concluding that providers avoid public programs because of "low reimbursement rates, administrative burden, and fear of being inundated with under-financed patients" ( $\mathrm{p}$. 10).

The lack of funding for adult dental care under Medicaid is injurious to the health and quality of life for individuals with developmental disabilities. Dental hygiene, preventive dental care, and acute dental care are all important to maintaining physical health. Not maintaining dental health is more costly because it takes its toll on a person's overall health and, ultimately, requires more expensive treatments, some of which may not be exclusively dental in nature and will, therefore, be charged to Medicaid. Cost-control is a factor in any decision about whether to provide Medicaid reimbursement, but blindness to the long-term costs of poor dental care is penny-wise but pound-foolish.

Further, it appears that all respondent families eventually found funding for covering out-of-pocket expenses, but not without undertaking extensive research into "free" funds and just being lucky. Undoubtedly, families could benefit from having one source from which they could learn about all potentially available funding sources. Such a source might be an " 800 " number at the state health agency or the professional societies of physicians and dentists, respectively.

\section{Appropriateness}

Our data (and the Report of the Surgeon General) indicate the need for improvements in physi- 
cian and dentist education regarding individuals with developmental disabilities. In particular, families reported unmet needs in dental care, orthodontic care, accessible equipment, and counseling. Because these issues were not our focus in this study, we recommend more research to pinpoint specific improvements and needs of families in auxiliary services.

The number of problems that exist within $\mathrm{OB} /$ GYN access for the small group of women with developmental disabilities in this study signifies a strong need for change. There is a delicate balance, however, between personal choice and education. Some parents have personal preferences for not ensuring that their daughter receives such care. Some of the preference, however, has been guided by a health care provider, either through misinformation or bias. It appears that the provider did not supply only objective information but, instead, imposed bias regarding the importance of such care. Preservice education and periodic inservice training opportunities seem warranted.

\section{Overall Health Care System}

Respondents suggested other improvements, none of which seem unreasonable. First, they wanted more information about their child's health status, especially when their child was younger. This desire can be satisfied through physician-dentist training and through the establishment, by the state physician and dental societies, and/or internet information-and-referral pages. Second, they wanted physicians and dentists to show greater concern and respect for individuals with developmental disabilities and their families. This is a matter for preservice and inservice education and could result from changes in Medicaid or other third-party reimbursement systems, where the changes would permit providers to spend the time with patients and families that is necessary to treat them with concern and respect rather than rushing them through treatment sessions. Finally, families still face shortages of providers who will accept Medicaid patients and who are sufficiently specialized to provide appropriate care.

Notwithstanding the shortcomings of health care (physician, dental, and OB-GYN) in Kansas for families who face the multiple issues of a family member with developmental disabilities, poverty or near-poverty, diversity, and rural residence, it seems that, at least in Kansas, health care is at an acceptable level. That finding contradicts the overall conclusion of the 2002 Report of the Surgeon General and much of the previous research. That finding, however, does not justify complacency.

\section{References}

Academy of Dentistry for Persons With Disabilities. (1996). Preservation of quality oral health care services for people with developmental disabilities: A position paper. Retrieved from http://www2. umdnj.edu/forumweb/dental.htm

Barnett, M. L., \& Ziring, P. (1988). Dental treatment program for patients with mental retardation. Mental Retardation, 26, 310-313.

Berg, B. L. (1998). Qualitative research methods for the social sciences. Boston: Allyn \& Bacon.

Bolden, A. J., Henry, J. L., \& Allkian, M. (1993). Implications of access, utilization, and need for oral health care by low income groups and minorities on the dental delivery system. Journal of Dental Education, 57, 888-898.

Brookins, G. K. (1993). Culture, ethnicity, and bicultural competence: Implications for children with chronic illness and disability. Pediatrics, 91, 1056-1062.

Brooks, R., \& Dwyer, R. (1997). Oral health and individuals with developmental disabilities: What community-based care and support providers think dentists and policy-makers need to know. Madison: University of Wisconsin, Health Promotion Project.

Burtner, A. P., \& Dicks, J. L. (1994). Providing oral health care to individuals with severe disabilities residing in the community: Alternative care delivery system. Special Care in Dentistry, 14, 188-193.

Caudle, P. (1993). Providing culturally sensitive health care to Hispanic clients. Nurse Practitioner, 18(12), 40, 43-51.

Chestnut, D. E. (1994). Perceptions of ethnic and cultural factors in the delivery of services in the treatment of sickle cell disease. Journal of Health and Social Policy, 5(3-4), 215-242.

Coffey, A., \& Atkinson, P. (1996). Making sense of qualitative data: Complementary research strategies. Thousand Oaks: Sage.

Connor, R. A., Hillson, S. D., \& Krawelski, J. E. (1995). Competition, professional synergism, and the geographic distribution of rural physicians. Medical Care, 33, 1067-1078.

Cornelius, L. J. (1993). Ethnic minorities and access to medical care: Where do they stand? Journal of the Association for Academic Minority Physicians, 4, 16-25. 
Crain, E. F., Kercsmar, C., Weiss, K. B., Mitchell, H., \& Lynn, H. (1998). Reported difficulties in access to quality care for children with asthma in the inner city. Archives of Pediatrics and Adolescent Medicine, 152, 333-339.

Damiano, P. C., Brown, E. R., Johnson, J. D., \& Scheetz, J. P. (1990). Factors affecting dentists' participation in a state Medicaid program. Journal of Dental Education, 54, 638-643.

Darling, R. B., \& Peter, M. I. (1994). Families, physicians, and children with special health needs: Collaborative medical education models. Westport, CT: Auburn House.

Davis, R. G., Fox, M., Johnston, J., Langner, B., McAdams, R., Moore, J., Redmond, T., McCunniff, M., \& Williams, K. (1999). Status and prospects for Medicaid beneficiaries' access to dental services in Kansas. Lawrence: University of Kansas.

Diamond, M., \& Zarafu, I. W. (1988). The role of the pediatrician in the care of the handicapped child. Pediatric Annals, 17, 738, 740-744.

Ebert, D. A., \& Heckerling, P. S. (1995). Communication with deaf patients. Knowledge, beliefs, and practices of physicians. Journal of the American Medical Association, 273, 227-229.

Fenton, S. (1993). Survey of training in the treatment of persons with disabilities. Interface, 9, 4.

Ferguson, F. S., Berentsen, B., \& Richardson, P. S. (1991). Dentists' willingness to provide care for patients with developmental disabilities. Special Care in Dentistry, 11, 234-237.

Fishman, B. M., Bobo, L., Kosub, K., \& Womeodu, R. J. (1993). Cultural issues in serving minority populations: Emphasis on Mexican Americans and African Americans. American Journal of the Medical Sciences, 306, 160-166.

Friedman, E. (1994). Money isn't everything: Nonfinancial barriers to access. Journal of the American Medical Association, 271, 1535-1538.

Giachello, A. L. (1998). Hispanic/Latino women's health. In L. A. Wallis (Ed.), Textbook of women's health (pp. 69-76). Philadelphia: Lippincott-Raven..

Giambruno, G., Cowell, C., Barber-Madden, R., \& Mauro-Bracken, L. (1997). The extent of barriers and linkages to health care for head start children. Journal of Community Health, 22, 101-114.

Grabois, E. W., Nosek, M. A., \& Rossi, D. R. (1999). Accessibility of primary care physicians' offices for people with disabilities. Archives of Family Medicine, 8, 44-51.

Harbaugh, C., \& Smith, T. (1998). Welfare reform and transportation: There is a connection. Public Roads, 61(4), 38-43.

Horner, R. D., Oddone, E. Z., \& Matchar, D. B. (1995). Theories explaining racial differences in the utilization of diagnostic and therapeutic procedures for cerebrovascular disease. Milbank Quarterly, 73, 443-462.

Hughes, D. C., Halfon, N., Brindis, C. D., \& Newacheck, P. W. (1996). Improving children's access to health care: The role of decategorization. Bulletin of the New York Academy of Medicine, 7, 237-254.

Jarrett, R. L. (1993). Focus group interviewing with low-income minority populations. In D. L. Morgan (Ed.), Successful focus groups: Advancing the state of the art (pp. 184-201). Newbury Park: Sage.

Kiefe, C. I., \& Hyman, D. J. (1996). Do public clinic systems provide health care access for the urban poor?: A cross-sectional survey. Journal of Community Health, 21, 61-70.

Kindig, D. A., \& Yan, G. (1993). Physician supply in rural areas with large minority populations. Health Affairs, 12, 177-184.

King, T. E., \& Brunetta, P. (1999). Racial disparity in rate of surgery for lung cancer. New England Journal of Medicine, 431, 1231-1233.

Knapp, K. K., Paavola, F. G., Maine, L. L., Sorofman, B., \& Politzer, R. M. (1999). Availability of primary care providers and pharmacists in the United States. Journal of the American Pharmaceutical Association, 39, 127-135.

Kogan, M. D., Kotelchuck, M., Alexander, G. R., \& Johnson, W. E. (1994). Racial disparities in reported prenatal care advice from health care providers. American Journal of Public Health, 84, 82-88.

Kopac, C. A., Fritz, J., \& Holt, R. A. (1998). Gynecologic and reproductive services for women with developmental disabilities. Clinical Excellence in Nurse Practitioner Practice, 2, 88-95.

Krueger, R. A. (1994). Focus groups: A practical guide for applied research. (2nd ed.). Thousand Oaks: Sage.

Lam, M., Riedy, C. A., \& Milgrom, P. (1999). Improving access for Medicaid-insured children: Focus on front-office personnel. JADA, 130, 365-373.

Lang, W. P., \& Weintraub, J. A. (1986). Comparison of Medicaid and non-Medicaid dental providers. Journal of Public Health Dentistry, 46, 207-211.

Levy, S. E., \& Hyman, S. L. (1993). Pediatric assessment of the child with developmental de- 
lay. Pediatric Clinics of North America, 40, 465477.

Marshall, C., \& Rossman, G. B. (1995). Designing qualitative research. Thousand Oaks: Sage.

McCubbin, H. I., Thompson, E. A., Thompson, A. I., McCubbin, M. A., \& Kaston, A. J. (1993). Culture, ethnicity, and the family: Critical factors in childhood chronic illnesses and disabilities. Pediatrics, 91, 1063-1070.

McEwen, E., \& Anton-Culver, H. (1988). The medical communication of deaf patients. Journal of Family Practice, 26, 289-291.

McKnight, H. C., Myers, D. R., \& Dushku, J. C. (1992). Method of payment for children's dental services by practice type and geographic location. Pediatric Dentistry, 14, 338-341.

Mort, E. A., Weissman, J. S., \& Epstein, A. M. (1994). Physician discretion and racial variation in the use of surgical procedures. Archives of International Medicine, 154, 761-767.

Nainar, S. M. H., Edelstein, B., \& Tinanoff, N. (1996). Access to dental care for Medicaid children in Connecticut. American Academy of Pediatric Dentistry, 18, 152-153.

Nainar, S. M. H., \& Tinanoff, N. (1997). Effect of Medicaid reimbursement rates on children's access to dental care. Pediatric Dentistry, 19, 315316.

Nelkin, V. S. R. S. M. (1996). Achieving healthy outcomes for children and families of diverse cultural backgrounds (Monograph for Health and $\mathrm{Hu}$ man Service Providers). Bernalillo: Southwest Communication Resources. (Available at $\mathrm{PO}$ Box 788, Bernalillo, NM 87004)

Newacheck, P. W., Hughes, D. C., \& Stoddard, J. J. (1996). Children's access to primary care: Differences by race, income, and insurance status. Pediatrics, 97(1), 26-32.

Nosek, M. A., Young, M. E., Rintala, D. H., Howland, C. A., Foley, C. C., \& Bennett, J. L. (1995). Barriers to reproductive health maintenance among women with physical disabilities. Journal of Women's Health, 4, 505-518.

O’Brien, K. (1993). Improving survey questionnaires through focus groups. In D. L. Morgan (Ed.), Successful focus groups: Advancing the state of the art (pp. 105-117). Newbury Park: Sage.

Palfrey, J. S., Samuels, R. C., Haynie, M., \& Cammisa, M. L. (1994). Health care reform: What's in it for children with chronic illness and disability. Journal of School Health, 64, 234-237.

Perkins, J. (1999). Overcoming language barriers to health care. Popular Government, 65(1).

Perkins, J., Simon, H., Cheng, F., Olson, K., \&
Vera, Y. (1998). Ensuring linguistic access in health care settings: Legal rights and responsibilities. Los Angeles: National Health Law Program.

Politzer, R. M., Harris, D. L., Gaston, M. H., \& Mullan, F. (1991). Primary care physician supply and the medically underserved: A status report and recommendations. Journal of the American Medical Association, 266, 104-109.

Rabinowitz, H. K., Diamond, J. J., Hojat, M., \& Hazelwood, C. E. (1999). Demographic, educational and economic factors related to recruitment and retention of physicians in rural Pennsylvania. Journal of Rural Health, 15, 212218.

Ralston, E., Zazove, P., \& Gorenflo, D. W. (1996). Physicians' attitudes and beliefs about deaf patients. Journal of the American Board of Family Practitioners, 9, 167-173.

Rask, K. J., Williams, M. V., Parker, R. M., \& McNagny, S. E. (1994). Obstacles predicting lack of a regular provider and delays in seeking care for patients at an urban public hospital. Journal of the American Medical Association, 271, 1931-1933.

Reichard, A., \& Turnbull, H. Rutherford, III. (2004). Perspectives of physicians, families, and case managers concerning access to health care by individuals with developmental disabilities. Mental Retardation, 42, 181-194.

Reichard, A., Turnbull, H. R., \& Turnbull, A. P. (1999). Including developmental disabilities in training programs for health professionals. Unpublished manuscript, University of Kansas, Lawrence.

Reichard, A., Turnbull, H. R., \& Turnbull, A. P. (2001). Dentist, family, and case manager perspectives on dental care for individuals with developmental disabilities in Kansas. Mental Retardation, 39, 268-285.

Riddick, S. (1998). Improving access for limited English-speaking consumers: A review of strategies in health care settings. Journal of Health Care for the Poor and Underserved, 9(Suppl.), S40-S61.

Rosenfeld, L. R. (1994). Your child and health care. Baltimore: Brookes.

Scott, F. (1990). A statewide survey of pediatricians on early identification and early intervention. Richmond: Virginia Department of Mental Health, Mental Retardation, and Substance Abuse Services, Part H Office.

Scott, F. G., Lingaraju, S., Kilgo, J. L., Kregel, J., \& Lazzari, A. (1993). A survey of pediatricians on 
early identification and early intervention services. Journal of Early Intervention, 17, 129138.

Seidel, J., Friese, S., \& Leonard, D. C. (1995). The ethnograph V4.0: A user's guide (Version 4.0). Amherst: Qualix Research Associates.

Simons, R. (1987). After the tears: Parents talk about raising a child with a disability. San Diego: Harcourt Brace.

Smith, M. W., Kreutzer, R. A., Goldman, L., CaseyPaal, A., \& Kizer, K. W. (1996). How economic demand influences access to medical care for rural Hispanic children. Medical Care, 34, 113118.

Stewart, D. W., \& Shamdasani, P. N. (1990). Focus groups: Theory and practice. Newbury Park: Sage.

Stiefel, D., Truelove, E., Martin, M., \& Mandel, L. (1997). Comparison of incoming dental school patients with and without disabilities. Special Care in Dentistry, 17, 161-168.

Taylor, S. J., \& Bogdan, R. (1998). Introduction to qualitative research methods: A guidebook and resource. New York: Wiley.

U.S. Department of Health an Human Services. (2002). Closing the gap: A national blueprint to improve the health of persons with mental retardation. Report of the Surgeon General's Conference on Health Disparities and Mental Retardation. Rockville: U.S. Department of Health and Human Services.

Umbarger, G. T., Turnbull, H. R., Morningstar, M. E., Reichard, A., \& Moberley, R. (2001). Funding challenges during transition to adulthood for young adults who use medical technology. Journal of Developmental and Physical Disabilities, $13,141-167$.

Vasquez, C., \& Javier, R. A. (1991). The problem with interpreters: Communicating with Spanish-speaking patients. Hospital and Community Psychiatry, 42, 163-165.

Venezie, R. D., \& Vann, W. F. (1993). Pediatric dentists' participation in the North Carolina Medicaid program. Pediatric Dentistry, 15, 175181.

Vittek, J., Winik, S., Winik, A., Sioris, C., Tarangelo, A., M., \& Chou, M. (1994). Analysis of orthodontic anomalies in mentally retarded developmentally disabled (MRDD) persons. Special Care in Dentistry, 14, 198-202.
Waldman, H. B. (1994). Distributing dentists throughout the nation. Annals of Dentistry, 53(2), 26-29.

Waldman, H. B. (1995). Rural and urban distribution of dentists, or Is there still gold in them thar hills? Illinois Dental Journal, 64, 121-125.

Waldman, H. B., Perlman, S. P., \& Swerdloff, M. (1998). What if dentists did not treat people with disabilities? Journal of Dentistry for Children, 65, 96-101.

Welner, S. L. (1998). Caring for the woman with a disability. In L. A. Wallis (Ed.), Textbook of women's health (pp. 87-92). Philadelphia: Lippincott-Raven.

Williams, D. R. (1999). Race, socioeconomic status, and health: The added effects of racism and discrimination. Annals of the New York Academy of Sciences, 896, 173-188.

Wilson, N. O. (1994). "My child needs special services": Parents talk about what helps ... . and what doesn't. Bedford, MA: Mills \& Sanderson.

Woloshin, S., Bickell, N. A., Schwartz, L. M., Gany, F., \& Welch, H. G. (1995). Language barriers in medicine in the United States. Journal of the American Medical Association, 273, 724-728.

Ziring, P. R., Kastner, T., Friedman, D. L., Pond, W. S., Barnett, M. L., Sonnenberg, E. M., \& Strassburger, K. (1988). Provision of health care for persons with developmental disabilities living in the community: The Morristown model. Journal of the American Medical Association, 260, 1439-1444.

Received 3/29/02, first decision 8/8/02, accepted 6/26/04

Editor-in-charge: Joseph Scotti

\section{Authors:}

Amanda Reichard, PhD (E-mail: areichard@khi. org), Senior Policy Analyst, Kansas Health Institute, 212 SW 8th St, Suite 300, Topeka, KS 66617. (This study was conducted when the senior author was affiliated with the Beach Center.) Therese Marie Sacco, MA, and $\mathrm{H}$. Rutherford Turnbull, III, LlB/JD, Co-Director, Beach Center on Disability, University of Kansas, Haworth Hall, 1200 Sunnyside Ave., Room 3136, Lawrence, KS 66045-7534.

This research was supported by a grant from the National Institute on Disability and Rehabilitation Research to the Beach Center on Disability, Grant \#H133B031133. 